\section{SAFETY AND EFFICACY OF NEOADJUVANT INTRAVESICAL ONCOLYTIC MV-NIS IN PATIENTS WITH UROTHELIAL CARCINOMA}

${ }^{1}$ Tanner Miest, ${ }^{2}$ Bradley Leibovich, ${ }^{3}$ Stephen Bardot, ${ }^{2}$ Paul Young, ${ }^{2}$ Stephen Boorjian, ${ }^{4}$ Mark Gonzalgo, ${ }^{2}$ Loren Herrera-Hernandez, ${ }^{2}$ Matthew Tollefson, ${ }^{2}$ Jeffrey Karnes, ${ }^{2}$ Paige Nichols, ${ }^{2}$ Tessa Kroeninger, ${ }^{3}$ Rachel Graham, ${ }^{4}$ Carole Lahana, ${ }^{5}$ Monica Reckner, ${ }^{2}$ Alysha Newsom, ${ }^{6}$ Nandakumar Packiriswamy, ${ }^{7}$ Janice Anoka, ${ }^{2}$ Kah Whye Peng, ${ }^{7}$ Erol Wiegert, ${ }^{7}$ Alice Bexon, ${ }^{6}$ Shruthi Naik* ${ }^{2}{ }^{1}$ University of Texas and Mayo Clinic, Houston, TX, USA; ${ }^{2}$ Mavo Clinic, Rochester, MN, USA; ${ }^{3}$ Ochsner Clinic, New Orleans, LA, USA; ${ }^{4}$ University of Miami, Miami, FL, USA; ${ }^{5}$ Vyriad, Inc., Rochester, MN, USA; ${ }^{6}$ Mayo Clinic and Vyriad Inc., Rochester, MN, USA; ${ }^{7}$ Vyriad Inc., Rochester, MN, USA

Background Bladder cancer is a leading cause of cancer death in the United States. ${ }^{1}$ The histology in $>90 \%$ of cases is urothelial carcinoma (UC). Tumors may present either as nonmuscle-invasive (NMIBC) or muscle-invasive disease (MIBC). Current standard of care for patients with high risk NMIBC includes transurethral resection of bladder tumor (TURBT) followed by intravesical immunotherapy with Bacillus CalmetteGuerin (BCG). ${ }^{2}$ Meanwhile, patients with BCG unresponsive NMIBC or MIBC are recommended to undergo radical cystectomy (RC), which adversely impacts quality of life and is associated with significant morbidity. ${ }^{3}$ MV-NIS is an investigational oncolytic measles virus with an excellent clinical safety profile. ${ }^{4}$ This ongoing phase I clinical study is designed to test the safety, efficacy and identify the recommended phase 2 dose (RP2D) of intravesical MV-NIS in patients with NMIBC or MIBC who are scheduled for RC and not eligible for neoadjuvant chemotherapy.

Methods Bladder UC patients were evaluated for eligibility and provided informed consent prior to enrolling. To date 8 patients have been enrolled: 4 to the single dose safety cohort, and 4 to the multi-dose expansion cohort. Patients were administered intravesical $\sim 1 \times 10^{9}$ TCID $_{50}$ MV-NIS once at least 1 week prior to RC (safety cohort), or twice at 4 and 2 weeks prior to RC (expansion cohort). Patients were closely monitored during the 2-hour instillation period. Tumor specimens from the pre-treatment TURBT and post-treatment RC were analyzed to determine pre- and post-treatment pathological stage and evaluate tumor killing and immune cell infiltrate. Results Intravesical MV-NIS treatment was well tolerated in all patients. Only a single Adverse Event (AE) attributable to MVNIS treatment (Grade 1 hematuria). AEs Grade $>2$ were related to post-surgical complications. Tumor pathology findings are summarized in table 1 . Tumor downstaging was observed in 4 of 8 patients. Among 4 patients in the expansion cohort, 2 had no residual disease (pT0). Central assessment of RC tissues showed significant inflammatory infiltrate in all treated bladder specimens. Detailed analyses are ongoing to characterize MV infection and immune infiltrate in bladder tissue

Conclusions The higher-than-expected rate of tumor downstaging and pT0 pathology, paired with the significant immune infiltrate observed in post-treatment bladder tissue, provide compelling evidence that intravesical MV-NIS has clinical activity against UC. These results support the use of two doses of $\sim 1 \times 10^{9}$ TCID $_{50}$ as the RP2D in future clinical studies for BCG unresponsive NMIBC or MIBC patients. MV-NIS induced inflammation may act synergistically with checkpoint blockade therapies.
Abstract 422 Table 1 Pre-treatment (TURBT) and post- treatment (RC) pathology

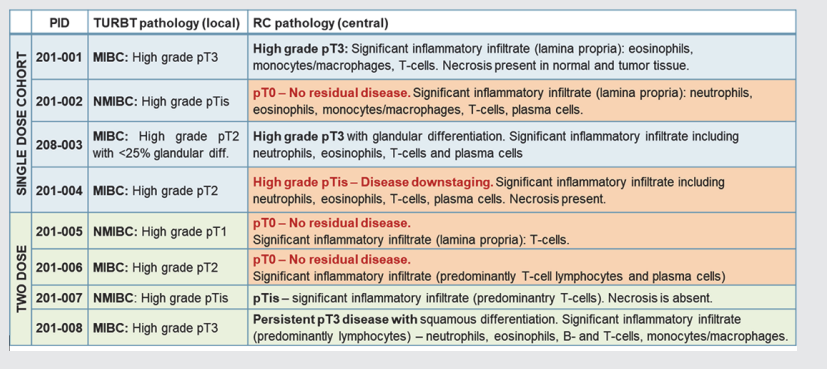

Trial Registration NCT03171493

\section{REFERENCES}

1. Siegel RL, Miller KD, Jemal A. Cancer statistics, 2019. CA Cancer J Clin 2019;69 (1):7-34.

2. Knowles MA, Hurst CD. Molecular biology of bladder cancer: new insights into pathogenesis and clinical diversity. Nat Rev Cancer 2015;15(1):25-41.

3. Zakaria AS, Santos F, Dragomir A, Tanguay S, Kassouf W, Aprikian AG. Postoperative mortality and complications after radical cystectomy for bladder cancer in Quebec: A population-based analysis during the years 2000-2009. Can Urol Assoc / 2014:8(7-8):259-267.

4. Galanis E, Atherton PJ, Maurer MJ, Knutson KL, Dowdy SC, Cliby WA, Haluska P $\mathrm{Jr}$, Long HJ, Oberg A, Aderca I, Block MS, Bakkum-Gamez J, Federspiel MJ, Russell SJ, Kalli KR, Keeney G, Peng KW, Hartmann LC. Oncolytic measles virus expressing the sodium iodide symporter to treat drug-resistant ovarian cancer. Cancer Res 2015;75(1):22-30.

Ethics Approval Approval was received from the Institutional Review boards (IRBs) at all clinical sites including Mayo Clinic (\#17-004167); Ochsner Health (\#2020 060); and University of Miami (\#20200174). All study participants are required to review and sign an IRB approved informed consent before taking part in the clinical trial.

http://dx.doi.org/10.1136/jitc-2021-SITC2021.422 